\title{
Die Festrede auf August Bier als Problem. Arthur Israel (1883-1969) und seine Auseinandersetzung mit der Berliner Chirurgischen Universitätsklinik
}

Wilfried Witte

\section{Summary}

Arthur Israel was associated with August Bier's University Surgical Clinic in Berlin from 1910 to 1933, in the end as the head of the downgraded (outpatient) clinic. After he was forced out of his Berlin post, Israel was department head in the Israelitisches Krankenhaus Hamburg until he emigrated to the U.S. in 1940, settling in New York. In 1960, he returned to Germany. Based on papers from Israel's estate, the article describes his life stations and his struggle to cope with his time at the Berlin university clinic. The focus is on a meeting in Berlin in 1961. His departure from the usual practice of heaping praise on the clinic and its former head marks the beginning of a neverpublished critique of conditions in the clinic. The ambivalence reflected in his evaluation is in stark contrast to the traditional cultivation of "Bier's School".

Keywords: Speech; Arthur Israel; August Bier; surgery; emigration; remigration; Ziegelstraße - "Bier's School”

\section{Zusammenfassung}

Arthur Israel war von 1910 bis 1933 an der Chirurgischen Universitätsklinik Berlin August Biers tätig, zuletzt als Leiter der verbliebenen (Poli-)Klinik. Nach der gewaltsamen Vertreibung aus dem Amt war er Chefarzt im Israelitischen Krankenhaus in Hamburg, bevor er 1940 in die USA (New York) emigrierte. Im Jahr 1960 kehrte er nach Deutschland zurück. Ausgehend von

Dr. med., MA, Institut für Geschichte der Medizin der Charité, Berlin; Klinik für Anästhesiologie und operative Intensivmedizin, Charité Campus Benjamin Franklin, Berlin (wilfried. witte@charite.de). 
einem schriftlichen Nachlass Israels beschreibt der Aufsatz Stationen seines Lebens und seine Auseinandersetzung mit der Zeit an der Berliner Universitätsklinik. Im Zentrum steht eine Zusammenkunft im November 1961 in Berlin. Sein Bruch mit der üblichen Praxis, Festreden auf die Klinik und deren ehemaligen Leiter zu publizieren, markiert den Beginn einer Kritik an Verhältnissen in der Klinik, die selbst nicht mehr veröffentlicht wurde. Die Ambivalenz, die dem zugrunde lag, widerstrebte der Traditionspflege einer «Schule» Biers.

\section{Die Lebensgeschichte eines Opfers der NS-Diktatur}

Das nationalsozialistische Regime setzte bekanntlich die deutsch-jüdischen Ärzte und Ärztinnen einer massiven Gewalt aus und führte Brüche in deren Leben herbei, die oft in der Ermordung endeten. In einem Akt der «Rücknahme der Emanzipation» ${ }^{1}$ wurde ihr Aktionsradius stufenweise minimiert oder unterbunden. Juristisch wurde dies u.a. umgesetzt durch das «Gesetz zur Wiederherstellung des Berufsbeamtentums» aus dem Jahre 1933, das sie aus dem öffentlichen Gesundheitswesen verbannte, sowie durch die 4. und 8. «Verordnung zum Reichsbürgergesetz» in den Jahren 1938 und 1939, durch die sie ihre Approbation verloren und zu jüdischen «Krankenbehandlern» degradiert wurden. ${ }^{2}$ Diese Maßnahmen waren die Grundlage dafür, die Ärzte und Ärztinnen zu Opfern der NS-Diktatur zu machen. Die Perspektive der Opfer nachzeichnen zu wollen, ist jedoch mit besonderen Schwierigkeiten verbunden.

Die Betroffenenperspektive stellt ein methodisches Problem in der Medizinhistoriographie dar. Beschäftigt man sich «aus heutiger Perspektive» mit den Verbrechen des Nationalsozialismus, so kann man den Opfern auf dem Weg der historischen Analyse emotional nicht gerecht werden. ${ }^{3}$ Außerdem wird «die Beschäftigung mit Betroffenen von NS-Verbrechen» dadurch erschwert, «dass die Quellenlage zu ihnen oft desolat ist, da sie mit ihrem Tod oder ihrer erzwungenen Emigration im Nebulösen endet». ${ }^{4}$ Häufig kommt das Verfahren der kollektiven Biographie zur Anwendung, das es ermöglichen soll, ein Personenkollektiv im gesellschaftlichen Zusammenhang zu erforschen, indem individuelle Lebensläufe vergleichend analysiert

\footnotetext{
1 Brenner 2008, 302-311.

2 Schwoch 2001; Jütte 2011.

3 Fangerau/Krischel 2011, 19.

4 Fangerau/Krischel 2011, 23.
} 
werden. ${ }^{5}$ Wie Anna von Villiez in Anlehnung an Thomas Etzemüller ausgeführt hat, handelt es sich bei einer kollektiven Biographie allerdings um ein Konstrukt, das erst «im Auge des Betrachters Form» gewinnt, so dass dargestellte Lebensläufe nicht als ein Faktum aufgefasst werden sollten. ${ }^{6}$ Dies erscheint insbesondere deshalb als relevant, weil die Tradition der Biographie in der Medizingeschichte ein «fester Bestandteil einer standeseigenen Identitätsbildung» ist, die darauf ausgelegt ist, die Verdienste der jeweiligen Mediziner zu würdigen. Um diesem tendenziell als unkritisch angesehenen Verfahren nicht zu folgen, hat sich Anna von Villiez in ihrer kollektiven Biographie «nicht arischer» Ärzte in Hamburg in den Jahren 1933 bis 1945 darum bemüht, die Brüche im Leben der von ihr behandelten Ärzte nicht diesem Narrativ zu unterwerfen. Ihre Monographie sollte nicht dazu dienen, die Lebensgeschichten der Hamburger Ärzte in der eigenen Perspektive allein danach zu strukturieren, dass es sich «um besonders verdiente Mitglieder der Gesellschaft» handelte, «die durch die Verfolgung im Nationalsozialismus um den ihnen zustehenden Platz in der Gesellschaft und in der Geschichtsschreibung der eigenen Zunft, um ihren «Status〉 gebracht worden seien». ${ }^{7}$ Das erlittene Unrecht, könnte man ergänzen, würde auch dann nicht geringer werden, wenn das Merkmal des besonderen Verdienstes nicht ausfindig gemacht werden könnte.

Arthur Israel (1883-1969), dem Arzt, um den es in diesem Aufsatz gehen soll, war sein Ansehen bei den deutschen Medizinern besonders wichtig. Aus Berlin stammend, war er lange Jahre an der Berliner Universitätsklinik unter August Bier (1861-1949) tätig, danach in Hamburg, bevor er in die USA emigrierte und gut ein Jahrzehnt nach dem Zweiten Weltkrieg nach Deutschland remigrierte. Auch sein Lebenslauf lässt sich bei der gegenwärtigen Quellenlage nur fragmentarisch wiedergeben. Gleiches gilt für seine Perspektive als Betroffener des NS-Regimes. Es sind jedoch Aussagen über seine späten Lebensjahre und über die Wandlung seiner Wahrnehmung des eigenen Lebens möglich. Die Darstellung muss sich deshalb nicht auf die passiv erlebten Brüche, die durch den Nationalsozialismus zustande kamen, beschränken.

Der Schwerpunkt des Aufsatzes liegt in der Beschreibung des bislang weitgehend unbekannten Lebensweges Arthur Israels. Es soll dabei nicht darum gehen, Israels in Vergessenheit geratene Verdienste zu würdigen. Der Fokus der Darstellung liegt auf der diachronen Perspektive des Protagonisten.

5 Schröder 1985.

6 Etzemüller 2003, 73; von Villiez 2009, 24.

7 von Villiez 2009, 25. 
Während Israel bis weit über den Zweiten Weltkrieg hinaus eine traditionelle Perspektive auf das prägende einstmalige Wirken und Leben in der Berliner Universitätsklinik einnahm, zeichnete sich ab 1961, in seinem letzten Lebensjahrzehnt, ein perspektivischer Traditionsbruch ab, der es ihm erlaubte, einen anderen Blickwinkel auszuloten. Das Bild, das sich ihm aus diesem Blickwinkel bot, blieb unscharf. Die Dissonanz, die sich allerdings darin kundtat, wird in der abschließenden Synopse im Sinne einer alternativen Lesart des typischen Lebensweges eines jüdischen Arztes und Wissenschaftlers in Deutschland in der ersten Hälfte des 20. Jahrhunderts hypothetisch gedeutet. Der Sehnsuchtsort der Klinik in der Ziegelstraße, der ihm einst durch die Nationalsozialisten entrissen worden war, scheint für den Protagonisten in der retrospektiven Betrachtung verblasst zu sein. Die Dissonanz führte zu Arthur Israels faktischer Ablehnung der Festrede auf Bier.

Als hauptsächliche Quellenbasis des Aufsatzes dient ein Nachlass Arthur Israels im Institut für Geschichte der Medizin Berlin, der - im Umfang eines Schubers - amtliche Dokumente, Briefe (vorrangig aus den 1960er Jahren) und andere Schriftstücke umfasst. ${ }^{8}$

\section{Der Vater, die Familie}

Chirurgie lag von Anfang an in der Luft, die er atmete, und sie dominierte sein Leben seit Anbeginn. So hat es Arthur Israels Kollege und früherer Mitarbeiter Hans Mannheim (1900-1972) im Jahr 1963 auf den Punkt zu bringen versucht, als er seinen ehemaligen Chef charakterisierte. Arthur Israels Umfeld war das des gehobenen Bürgertums in «pluralistischer Urbanität» ${ }^{9}$ gewesen; das Leben entsprach der Vorstellung einer «Erfolgsgeschichte» des deutschen Judentums im 19. und zu Beginn des 20. Jahrhunderts. ${ }^{10}$ Arthur Israel, geboren am 25. April 1883, wuchs in einer etablierten bürgerlichen Familie im Berliner Bezirk Tiergarten auf. ${ }^{11}$ Sein Vater, James Adolph Israel (1848-1926), war zu dieser Zeit ein gemachter Mann. Selbst aus einer anglophilen Berliner Familie stammend, hatte er seine medizinische Laufbahn bei dem Universitäts-Chirurgen Bernhard von Langenbeck (1810-1887) begonnen. Im Jahre 1881 konnte er die Leitung der Chirurgischen Abteilung des Jüdischen Krankenhauses Berlin übernehmen. Unter James Israel wurde das Jüdische Krankenhaus zu einer «international ange-

8 Nachlass.

9 van Rahden 2004, 362.

10 Rürup 1991.

11 Mannheim 1963. 
sehenen Musteranstalt, in der die neue Disziplin der urologischen Chirurgie gelehrt wurde». ${ }^{12}$ Außerdem beschäftigte er sich speziell mit der plastischen Chirurgie. Eine akademische Karriere war James Israel als Jude versagt, den Professorentitel erhielt er ehrenhalber im Jahr 1894. Sein internationales Renommee bezeugte eine Reise nach Konstantinopel im Jahr 1915, als er den osmanischen Sultan Mohammed V. behandelte. ${ }^{13}$

Das Ehepaar James Israel und Meta Israel, geb. Goldstein (1854-1930), hatte vier Kinder, zwei Töchter und zwei Söhne. Charlotte Israel heiratete einen früheren Assistenten ihres Vaters, Dr. Siegfried Levi (1877-1944), der im Konzentrationslager Theresienstadt ermordet wurde. ${ }^{14}$ Sie selbst «ging in den Gaskammern von Auschwitz zugrunde». ${ }^{15}$ Sie hatten zumindest einen Sohn (Rolf?), der in den 1960er Jahren in der Schweiz (Neuchâtel) als Psychiater tätig gewesen ist. ${ }^{16}$ Auch Else Israel heiratete, in zweiter Ehe, einen ehemaligen Assistenten ihres Vaters, Dr. Arthur Block, der 1943 im besetzten Belgien von der SS gehenkt worden ist. Nach der Befreiung wanderte sie mit ihrem Sohn Peter in die USA aus. ${ }^{17}$ Arthur Israel gab später in einem persönlichen Schreiben zu Protokoll: «Alle Mitglieder meiner Familie, die in Deutschland zurückgeblieben waren [,] sind elendiglich gestorben.» ${ }^{18}$

Die Söhne Wilhelm (1881-1959) und Arthur (1883-1969) sind Chirurgen geworden. Der ältere, Wilhelm, ist 1908 approbiert und promoviert worden. Er ist u.a. von seinem Vater am Jüdischen Krankenhaus zum (Plastischen) Chirurgen und Urologen ausgebildet worden. Mit ihm zusammen hat er 1925 ein urologisches Lehrbuch herausgegeben. ${ }^{19}$ Wilhelm Israel wurde am Jüdischen Krankenhaus zum Dirigierenden Arzt ernannt und war - von 1919 bis 1934 - als Privatdozent für Chirurgie an der Berliner Universität tätig. Dem endgültigen Verlust seiner Kassenzulassung in Berlin im Jahr 1937 ist er bereits im Dezember 1933 zuvorgekommen, indem er nach London übersiedelte. Dort war er als Chirurg tätig, seit 1939 als «specialist» gemeldet, 1947 erhielt er die britische Staatsangehörigkeit. ${ }^{20}$ Der Nachlass Arthur Israels enthält eine Heiratsanzeige (ohne Angabe eines Datums, vermutlich aus den frühen 1950er Jahren) Dr. William I. Israels mit einer Else Meyer, geb.

12 Winau/Vaubel 1983, 42.

13 Israel 2006.

14 Schwoch 2009, 519.

15 Schreiben Arthur Israels vom 16.10.1949 an einen Herrn Benölken, aus den USA, Nachlass.

16 Briefwechsel Arthur Israel und Albert Salomon, Nachlass.

17 Hartung von Doetinchem/Winau 1989, 107; Bloch/Israel/Schultze-Seemann 1983; Winau 1987; Israel 2006.

18 Schreiben Arthur Israels vom 16.10.1949 an einen Herrn Benölken, aus den USA, Nachlass.

19 Israel/Israel 1925.

20 Schwoch 2009, 381-382. 
Rothenberg. Die Trauung fand in London statt. ${ }^{21}$ Anders als sein Bruder hat Arthur Israel nicht geheiratet. Zumindest von 1960 bis 1969 ging er jedoch nicht allein durch das Leben, sondern in Begleitung eines «Frl. Solbrig», der aus New York stammenden Margaret A. Solbrig. 22

Anders als sein Bruder ist Arthur Israel auch nicht durch die chirurgische Schule seines Vaters gegangen, sondern durch diejenige August Biers (1861-1949).

\section{Arthur Israel in August Biers Klinik in der Ziegelstraße}

Nach dem Abitur im Jahre 1901 studierte Arthur Israel in Berlin, München, Freiburg im Breisgau und Straßburg im Elsass Medizin. Ein Jahr nach seinem Staatsexamen legte er 1907 seine Doktorarbeit vor, in der er erstmals das Krankheitsbild des nicht durch Nierenkrankheiten bedingten Bluthochdrucks (essentielle Hypertonie) klinisch beschrieb. ${ }^{23}$ Danach war er für einige Monate in der Inneren Klinik Ludolf von Krehls (1861-1937) in Straßburg tätig. Dem folgte eine halbjährige Beschäftigung im Pathologischen Institut des Städtischen Krankenhauses im Friedrichshain in Berlin bei Ludwig Pick (1868-1944). Von 1908 bis 1910 war er als Assistent im Institut für Infektionskrankheiten in Berlin (dem späteren Robert-Koch-Institut) unter August von Wassermann (1866-1925) in der experimentellen Therapie und Biochemie tätig. Wassermann schrieb 1920 in seinem Zeugnis: «Herr Dr. Israel verließ uns, weil er mit dem Aufenthalt am Institut nur ein gründliches wissenschaftliches Eindringen in die für sein eigentliches Fach, die Chirurgie, wichtige Hilfswissenschaft der Bakteriologie und experimentellen Therapie bezweckte. ${ }^{24}$ Ab 1908 war Israel Mitglied der Berliner medizinischen Gesellschaft. Zwei Jahre später, als seine Zeit in der Berliner Chirurgischen Universitätsklinik in der Ziegelstraße begann, wurde er Mitglied der Deutschen Gesellschaft für Chirurgie und der Berliner Gesellschaft für Chirurgie. ${ }^{25}$

August Bier wurde trotz aller persönlichen Gegensätzlichkeit zum Drehund Angelpunkt in Arthur Israels Leben. Charakterlich stach der als eher vornehm und feinsinnig beschriebene Israel von dem aus einer ländlichen Beamtenfamilie stammenden August Bier stark ab. Bier liebte die direkte,

21 Nachlass.

22 Nachlass; Staatsarchiv Hamburg Bestand 611-11?, Sig. 38 (2).

23 Israel 1907.

24 August von Wassermann (Kaiser Wilhelm-Institut für experimentelle Therapie, Berlin-Dahlem, 29. Juli 1920): Zeugnis, Nachlass.

25 Nachlass; Schwoch 2011, 222. 
geradlinige Ansprache, die auch vor Derbheiten nicht zurückschreckte. ${ }^{26}$ In einer Zeit, als es noch keine Anästhesiologie in Deutschland gab, hatte Bier seinen Ruhm mit einer technischen Neuerung zur Schmerzausschaltung bei Operationen, der Lumbalanästhesie, begründet. ${ }^{27}$ Erprobt in Selbstversuchen trug gerade das Moment der «heroischen Medizin» dazu bei, Biers exzeptionellen Ruf zu begründen. ${ }^{28}$

Im Jahr 1907 übernahm Bier die Klinik in der Ziegelstraße. Seine zahlreichen Privatpatienten bescherten ihm ein Vermögen, das es ihm erlaubte, 1912 das Gut Sauen bei Beeskow zu erwerben. Politisch war er deutschnational (und monarchistisch) ausgerichtet. Die Einführung des Stahlhelms beim Deutschen Militär geht auf sein Konto. Für den Sport setzte er sich nach dem Ersten Weltkrieg nicht zuletzt zur Steigerung der «Wehrertüchtigung» ein. ${ }^{29}$ Gleichzeitig mit dem Chirurgen der Charité, Ernst Ferdinand Sauerbruch (1875-1951), erhielt der Emeritus Bier im September 1937 auf dem Nürnberger Parteitag der NSDAP den «Deutschen Nationalpreis für Kunst und Wissenschaft» - als NS-Alternative zum Nobelpreis. ${ }^{30}$

Biers theoretischer Zugang zur Chirurgie war philosophisch grundiert. Er kreiste um eine neohippokratische Teleologie, die ein umstrittenes Votum für eine homöopathische Pharmakotherapie integrierte. ${ }^{31}$ Grundgedanke der Teleologie war die Zweckmäßigkeit als Betrachtungsweise, «die auf der Erfahrung fußt, dass im allgemeinen Bau und Leistungen des menschlichen Körpers zweckmäßiger Art sind». ${ }^{32}$ Naturphilosophie wurde neben Naturwissenschaft gestellt. Der Neohippokratismus betonte den Wert der Erfahrung des guten Arztes im Lichte der Philosophie Heraklits und «im Windschatten» des sogenannten Dritten Humanismus. ${ }^{33}$ Der Dritte Humanismus war eine Strömung in der Weimarer Republik, die «als Reaktion auf die durch die Novemberrevolution in Frage gestellten überkommenen Traditionen aus dem Kaiserreich (...) in der Vermittlung der Kenntnisse über die Antike an eine Bildungselite die Möglichkeit einer von diesem Bürgertum für notwendig gehaltenen sittlichen Erneuerung sah». ${ }^{34}$ Nach seiner aktiven Zeit als Chirurg verfasste Bier ein populärwissenschaftliches Buch, Die Seele, in dem er rezente Strömungen der Psychologie kritisierte. ${ }^{35}$ Nach-

26 Vogeler 1962, 328.

27 Winau 1987; Marx 1994; Goerig/Schulte am Esch 2012, 166-167.

28 Domrich 1940.

29 Keil 2006.

30 Goerig/Schulte am Esch 1999.

31 Bier 1922; Doms 2004.

32 Bier 1910, 17.

33 Lammel 1998, 200.

34 Harig 1986, 1407.

35 Bier 1939ff.; vgl. Henschen 1951. 
gelassene Schriften wurden unter dem Titel «Das Leben» 1951 herausgegeben. ${ }^{36}$

Israel durchlief bis 1931 mustergültig alle Stufen in der Klinik in der Ziegelstraße. Im Jahre 1913 wurde er planmäßiger erster klinischer Assistent, von 1914 bis 1916 leitete er Krankenstationen. Vom Februar 1917 bis Dezember 1918 war er als landsturmpflichtiger Arzt «im Felde». Im Jahr 1923 folgte die Habilitation, ab dem 3. März 1923 war er Privatdozent in der Medizinischen Fakultät der Universität Berlin. ${ }^{37}$ Vom 26. März 1928 bis zum 22. Oktober 1935 führte er den Titel eines nichtbeamteten außerordentlichen Professors für das Fach Chirurgie in Berlin. Ab dem 15. April 1931 war er Oberarzt der Klinik, ${ }^{38}$ vom 31. März $1932^{39}$ bis zum 4. April $1933^{40}$ Leiter der verbliebenen Poliklinik. Laut Hans Mannheim war sein Ruf tadellos, er habe sich nicht an Intrigen beteiligt, sondern galt als das «gute Gewissen» der Klinik («The clinic's good conscience»). ${ }^{41}$

\section{Die Klinik in der Ziegelstraße nach 1931}

Anfang Oktober 1931 war Bier die Schließung der Klinik in der Ziegelstraße, die ursprünglich von Sauerbruch als Universitätsklinik übernommen werden sollte, mitgeteilt worden. Es verblieb lediglich die Poliklinik. Sauerbruch erhielt einstweilen das Direktorat für die Poliklinik, Israel wurde der ärztliche Leiter. Damit übernahm Arthur Israel die Bier'sche Klinik, soweit sie erhalten blieb, von seinem akademischen Lehrer. Ihm zur Seite standen u.a. der planmäßige Assistent Constantin von Bramann (1900-1989) und der außerplanmäßige Assistent Karl Hermann Domrich (1901-1989). ${ }^{42}$ Domrich trat am 1. Mai 1933 der NSDAP bei und verblieb in der Klinik in der Zeit des Nationalsozialismus, von Bramann verließ die Klinik.

An die Macht gekommen, beendeten die Nationalsozialisten Israels Zeit in der Ziegelstraße umgehend. Israel wurde vertrieben. ${ }^{43}$ August Bier nahm dies am 28. April 1933 als eine Folge der «politischen Umwälzung» bedauernd zur Kenntnis. ${ }^{44}$

36 Bier 1951.

37 Humboldt-Universität zu Berlin, Archiv, Med. Fak. Nr. 1357 - Habilitationen 1922-1924, Arthur Israel, Bl. 175-185.

38 Lammel 1993, 65.

39 Lammel 1994, 573.

40 Schreiben Arthur Israels vom 16.10.1949 an einen Herrn Benölken, aus den USA, Nachlass.

41 Mannheim 1963.

42 Lammel 1994, 575-576.

43 Vgl. Schagen 2008.

44 Schreiben August Biers (Berlin) an Fritz Warburg (Hamburg), 28.4.1933, Nachlass. 
Arthur Israel stellte die Vorgänge 1949 wie folgt dar:

Nach der üblichen Zwischenzeit von fünf Jahren wurde ich 1928 Professor, wurde bald darauf klinischer Oberarzt und 1932, nachdem mein Chef Bier sich zurückgezogen hatte, stellvertretender Leiter der Klinik und Poliklinik. Die Freude dauerte nur ein Jahr, denn am 4. April 1933 wurde ich in der Klinik von den Nazis verhaftet und für 24 Stunden im Braunen Hause eingesperrt. Das war mein Abgang aus der Klinik (,) in der ich 23 Jahre gearbeitet hatte. ${ }^{45}$

Im Rahmen der Verhandlungen des «Bundesergänzungsgesetzes zur Entschädigung für Opfer der nationalsozialistischen Verfolgung (BEG)» ergänzte Israel Ende 1953:

Wir [sein Assistent Hans Mannheim und er] wurden nach dem Braunen Haus in der Prinz Albrechtstrasse gebracht und gezwungen, einen Verzicht auf unsere Stellung bzw. einen Antrag auf Beurlaubung zu unterschreiben. Nach 24 Stunden wurden wir wieder entlassen. Wir durften die Klinik nicht mehr betreten und die offizielle Kündigung erfolgte ein halbes Jahr später $(. . .)^{46}$

Was folgte, war nicht nur der Rufmord an Israel, sondern die Tilgung seines Namens. Die Klinik wurde durch die Nationalsozialisten ideologisch in Beschlag genommen. Im November 1933 wurde der «linientreue» Unfallchirurg Georg Magnus (1883-1942) aus Bochum als neuer Lehrstuhlinhaber berufen. Einer seiner Assistenten und (seit dem 1. April 1934) Oberarzt war Karl Brandt (1904-1948), der ab dem 14. Juni 1934 zugleich als Adolf Hitlers Begleitarzt fungierte. ${ }^{47}$ Brandt selbst schrieb 1939 über die Zeit nach dem Austritt von August Bier aus der Klinik am 31. März 1933:

Um den gegebenen lokalen Anforderungen zu genügen, musste im Gebäudekomplex der Ziegelstraße eine Rumpfpoliklinik erhalten bleiben (...) Der Leiter, Professor Vogeler, selbst ein langjähriger Schüler und Mitarbeiter Biers, war bestrebt, wenigstens die Erinnerung an die frühere große Klinik bei Patienten und in Ärztekreisen wach zu halten. ${ }^{48}$

Das sah auch Vogeler so - zumindest ist das seiner eigenen Darstellung in der von ihm verfassten Biographie Biers von $1941 \mathrm{zu}$ entnehmen. ${ }^{49}$ Karl Vogeler (1889-1978), der «bis zum 31. März 1932 ebenfalls planmäßiger Assistent in der Ziegelstraße gewesen war, am 13. März 1932 auf Drängen Biers hin vorzeitig zum n.b.a.o. Professor ernannt und am 10. Dezember 1932 der SA und im Mai 1933 der NSDAP beitrat», war durch das Ministerium am 3. Mai 1933 beauftragt worden, die Leitung zu übernehmen, bevor Magnus das Ruder übernahm. ${ }^{50}$

45 Schreiben Arthur Israels vom 16.10 .1949 an einen Herrn Benölken, aus den USA, Nachlass. 46 Arthur Israel, Schilderung des Verfolgungsvorgangs, 20.11.1953. In: Staatsarchiv Hamburg Bestand 351-11, Sig. 6711.

47 Schmiedebach/Schwoch 2011.

48 Brandt 1939, 187.

49 Vogeler 1942, 98.

50 Lammel 1994, 574. 
Wie Brandt drei Jahre zuvor vermied Vogeler die Erinnerung an die Zeit von Israels ca. einjähriger Leitung. Auch dessen vormalige Bedeutung in der Klinik unterschlug er. Als sich Biers aktive Zeit schon dem Ende zugeneigt hatte, hatten die Feierlichkeiten zu Biers 70. Geburtstag stattgefunden, u.a. im Hörsaal der Klinik. Die Festrede halten zu dürfen, war ein Verdienst, das Arthur Israel zukam. Am 24. November 1931 sprach Israel in typischer Diktion u.a. folgende Worte:

(...) Hier ist Ihre und unsere kleinere intimere Welt. Dieser Raum ist gar nicht zu denken ohne Ihre Persönlichkeit und Ihre Persönlichkeit nicht ohne diesen Raum. Darum möchten wir Ärzte der Klinik Ihnen an dieser Stätte unsere Glückwünsche wiederholen, aber zugleich auch unseren Dank abstatten für alles, was Sie uns gewesen sind, und was Sie uns, so hoffen wir, noch sein werden. Die Mitwelt dankt Ihnen für Ihre Werke, die Nachwelt wird Ihnen diesen Dank bewahren, die Kranken danken dem Arzte, wir aber danken unserem Lehrer und Führer. Ihr Wesen hat uns immer beherrscht, doch nie regiert $(. . .)^{51}$

Vogeler, der 1934 die Klinik verließ und Chefarzt in Stettin wurde, hat in seiner Biographie Biers von 1941 Israels Autorenschaft verschwiegen und stattdessen lediglich einen «Assistenten der Klinik» erwähnt. ${ }^{52}$ Domrich hat dieses Verhalten noch 1963 damit gerechtfertigt, dass der Nationalsozialismus es damals «verlangte». ${ }^{53}$ Wie weit dies reines Mitläufertum war oder politischer Überzeugung entsprach, bleibt dahingestellt.

\section{Hamburg und die Emigration}

Israel sah sich 1933 nach einer neuen Betätigungsstätte um. Er bewarb sich auf die freigewordene Stelle des Leiters der Chirurgischen Abteilung des Krankenhauses der Deutsch-Israelitischen Gemeinde Hamburg (Israelitisches Krankenhaus) im Hamburger Stadtteil St. Pauli. Kuratoriumsvorsitzender des traditionsreichen Hauses war der wohlhabende Mitinhaber des Bankhauses M.M. Warburg \& Co. in Hamburg, Dr. Fritz M. Warburg (1879-1964). Vier Wochen nach der Bewerbung konnte Israel die Stelle antreten. ${ }^{54}$

51 Vogeler 1942, 56-57.

52 Vogeler 1961.

53 Domrich 1963, 495.

54 Fritz Warburg, Zeugnis des Krankenhauses der Deutsch-Israelitischen Gemeinde Hamburg für Arthur Israel, 8. Dezember 1938, Nachlaß; Schreiben Arthur Israels vom 16.10.1949 an einen Herrn Benölken, aus den USA, Nachlass. 


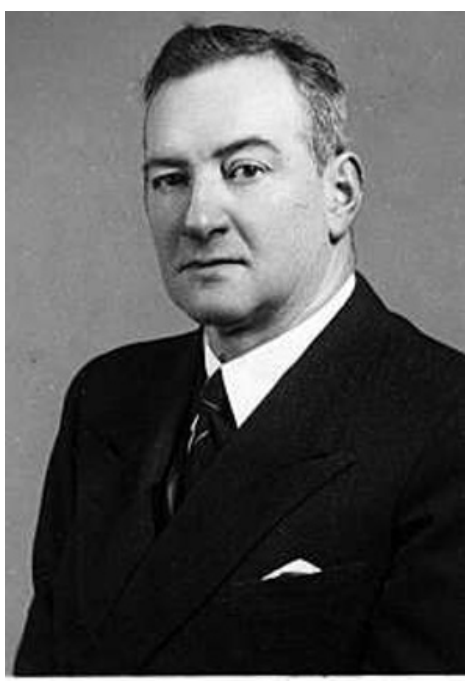

Arthur Isreal in seiner Hamburger Zeit, Nachlass Arthur Israel, Institut für Geschichte der Medizin der Charité Berlin.
Seit dem 10. Juli 1933 war er bei der Polizeibehörde Hamburg gemeldet. Die Verlegung des Wohnsitzes von Berlin nach Hamburg wurde vom preußischen Wissenschaftsministerium zunächst genehmigt, im August 1935 jedoch vom Reichs- und Preußischen Wissenschaftsministerium untersagt. Damit waren auch Israels Versuche, weiter als Professor Vorlesungen an der Universität Berlin abzuhalten, unterbunden. ${ }^{55}$ Die legalistische Argumentation war perfide. Am 22. Oktober 1935 schrieb das Wissenschaftsministerium an die Medizinische Fakultät Berlin: «Nachdem der n.b.a.o. Professor Dr. Artur Israel erklärt hat, außerstande zu sein, seinen Wohnsitz nach Berlin zurückzuverlegen und eine Beurlaubung aus grundsätzlichen Erwägungen nicht in Frage kommt, ist die venia legendi vom Wintersemester als erloschen anzusehen.» Am darauf-

folgenden Tag beurlaubte der Rektor der Universität Berlin Israel. Am 25. Oktober teilte die Medizinische Fakultät dem Rektor mit, dass Israels Venia Legendi erloschen sei. Am 22. Februar 1936 verkündete die FriedrichWilhelms-Universität Berlin, dass allen jüdischen Professoren rückwirkend zum 31. Dezember 1935 die Lehrbefugnis entzogen werde. ${ }^{56}$

Israel erlebte die Zeit in Hamburg nichtsdestotrotz eigenen Bekundungen zufolge bis 1937 als «sehr schön» und «befriedigend». ${ }^{57}$ Im Mai 1938 übernahm er auch noch das Direktorat des Krankenhauses. Die Lage wurde jedoch auch für ihn zusehends brenzliger und ging mit der Degradierung zum «Krankenbehandler» einher. ${ }^{58}$

Wenige Tage vor der «Reichskristallnacht» (9./10. November 1938) kehrte Fritz Warburg aus Stockholm zurück, wo er nach einer Lösung der schwierigen finanziellen Lage des Krankenhauses gesucht hatte. Er wurde mehrere Monate inhaftiert. Nach seiner Entlassung aus der Haft emigrierte er im Mai 1939 nach Schweden. ${ }^{59}$ Max Warburg (1867-1946), sein Bruder und Mitinhaber der Bank, war bereits 1938 in die USA emigriert. Seit 1933 hatte

55 In Hamburg hielt Israel Fortbildungskurse für jüdische Ärzte ab, Nachlass.

56 Humboldt-Universität zu Berlin, Archiv, UK Personalia, I 021, Arthur Israel.

57 Schreiben Arthur Israels vom 16.10.1949 an einen Herrn Benölken, aus den USA, Nachlass; Brandmann 1997, 70-71.

58 Vgl. Schwoch 2011.

59 Lorenz 1997, 74-76. 
Max Warburg als Vorsitzender des «Hilfsvereins der Juden in Deutschland» jüdischen Emigranten geholfen, durch Vermögenstransfers nach der Emigration teilweise ihre Besitzstände zu erhalten. Bevor sie das Land verließen, gründeten die Brüder Warburg noch das «Sekretariat Warburg», das von 1938 bis 1941 unter der Leitung des jüdischen Bankiers Robert Solmitz (1894-1982) stand. ${ }^{60}$ Das «Sekretariat» versuchte laut Dorothea Hauser, «die weitverzweigten Verbindungen der inzwischen «arisierten〉 Warburg-Bank für den Vermögenstransfer jüdischer Emigranten ins Ausland nutzbar zu machen, was ab 1940 kaum noch möglich war.» ${ }^{61}$

Israel quälte sich mit der Entscheidung, Deutschland zu verlassen, und zögerte lange. ${ }^{62}$ Als er schließlich im Juli $1940^{63}$ Hamburg verließ, konnte er sich auf die Unterstützung des Warburg-Netzwerks stützen. Die Emigration führte ihn wie die meisten seiner Kollegen, die ebenfalls vor den Nazis fliehen mussten und die nicht versuchten, nach Palästina zu gelangen, in die USA, und zwar nach New York. ${ }^{64}$ Von Amsterdam aus halfen ihm dabei Max' Sohn Erich M. Warburg (1900-1990) und von Hamburg aus Robert Solmitz. In New York nahm Israel sogleich Kontakt auf zu Max Warburg. ${ }^{65}$ Bereits im Oktober 1939 war es gelungen, für Arthur Israel wenigstens 2000 US-Dollar in New York zu deponieren. ${ }^{66}$ Der weitaus größte Teil seines Besitzes blieb in Deutschland zurück. ${ }^{67}$

Israels Reise führte ihn über die Sowjetunion via Sibirien nach Japan und dann per Schiff an die Westküste der USA, nach Seattle. Von dort ging es auf dem Landweg weiter zum Bestimmungsort New York. ${ }^{68}$ In New York musste er beruflich wieder bei null anfangen. ${ }^{69}$ Bis zum 15 . Oktober 1936 war in New York die deutsche medizinische Approbation anerkannt worden. Die Regelung kam für Israel jedoch nicht mehr zum Tragen: zum einen, weil er später emigrierte, zum anderen, weil ihn die NS-Machthaber unterdessen zum «Krankenbehandler» gestempelt hatten. ${ }^{70}$ «Die Umstellung war nicht so einfach. Die nicht leichten Examina erforderten sehr viel Zeit und Mühe»,

$60 \mathrm{http} / /$ www.grossborstel.de/index.php/content/view/342/103/ (Zugriff 18.6.2012).

61 Fischer 2009; vgl. Hauser 2010.

62 Schreiben Wilhelm Israels an Arthur Israel vom 3.8.1939, Nachlass.

63 Lorenz 1997, 78.

64 Pearle 1984; Baader 1984; Kröner 1989; Damskis 2009.

65 Schreiben von Erich M. Warburg an Arthur Israel, Amsterdam, 17.08.1939, Nachlass;

Schreiben von Robert Solmitz an Arthur Israel, Hamburg, 4.7.1940; Schreiben von Max M.

Warburg an Arthur Israel, New York, 26.12.1940, Nachlass.

66 Telegramm Deutsche Reichspost vom 3.10.1939, Nachlass.

67 Staatsarchiv Hamburg Bestand 351-11, Sig. 6711.

68 Nachlass; Schreiben der Hamburg-Amerika Linie Hamburg an Firma M.M. Warburg \& Co. vom 8.3.1941, Staatsarchiv Hamburg Bestand 351-11, Sig. 6711.

69 Vgl. Jacob 2012.

70 von Villiez 2009, 132, 299. 
schrieb er selbst über diese Zeit. ${ }^{71}$ Im Oktober 1942 wurde er in New York als «Physician and Surgeon» registriert. ${ }^{72}$ Seine Lehrbefugnis war durch die Annullierung im nationalsozialistischen Deutschland auch in den Vereinigten Staaten passé.

Israel ließ sich als Chirurg nieder. Seine medizinischen Betätigungen in New York garantierten den Unterhalt in bescheidenen Verhältnissen. Ab 1943 hatte Israel ein «staff appointment» am Beekman Hospital ${ }^{73}$ in Lower Manhattan und beim Mount Sinai Hospital an der Fifth Avenue. Im Mount Sinai Hospital wurde er als «clinical assistant» erstmalig am 29. März 1943 für ein Jahr eingestellt. Sein Tätigkeitsbereich war das «OutPatient Department, Genito-Urinary Division», wo er dreimal in der Woche, jeweils nachmittags, arbeitete. Das «appointment» wurde jährlich verlängert. Am 5. Januar 1948 stieg er zum «Senior Clinical Assistant» auf, am 30. April 1950 zog er sich - mit Erreichen des 67. Lebensjahrs - von der klinischen Arbeit in der Klinik zurück. ${ }^{74}$ Seine Einnahmen waren gering und umfassten bis in die 1950er Jahre «kaum die Hälfte des Durchschnittseinkommens eines amerikanischen Arztes in gleicher Stellung». ${ }^{75}$ Das Ansinnen, nach Israel überzusiedeln, hat Arthur Israel abgelehnt. ${ }^{76}$

Im Jahr 1944 wurde Israel zwar Mitglied von wenigstens zwei medizinischen Vereinigungen, der «Rudolf Virchow Medical Society in the City of New York», die seit 1933 großen Zulauf von Emigranten erhielt,${ }^{77}$ und des «International College of Surgeons». Im Exil ging es jedoch vorrangig ums reine Überleben. Die Zeit im Exil setzte seinem «Gemüt» zu. Erst nach der Remigration habe er sich wieder - eigenem Bekunden aus dem Jahre 1967 zufolge - «viel aufgeschlossener für menschliche Beziehungen» gefühlt, als er es «in New York sein konnte».78

71 Schreiben Arthur Israels vom 16.10.1949 an einen Herrn Benölken, aus den USA, Nachlass.

72 Originalurkunde im Nachlass.

73 Heute das New York Downtown Hospital.

74 George B. Bernheim (The Mount Sinai Hospital), Schreiben an Arthur Israel, New York, 14. April 1943, in: Nachlass; Medical Staff Office: Personalkartei, Karte zu «Israel, Arthur, MD», Archives of the Mount Sinai Hospital New York.

75 Laut einer Übersicht von New Yorker Wirtschaftsprüfern (Heinemann \& Spitzer) aus dem Jahre 1953 verdiente er von 1943 bis 1952 von 1200 bis 3300 US-Dollar, im Schnitt 2550 US-Dollar per annum (Schriftwechsel Dr.J.Auerbach, Consultant on International Law, London, mit dem Amt für Wiedergutmachung Hamburg in den Jahren 1953/54, Staatsarchiv Hamburg Bestand 351-11, Sig. 6711).

76 Schreiben von Dr. Hanna Hirsch (Petach Tikwa, Israel) vom 19.8.1950 an Arthur Israel,Nachlass.

77 Berberich/Lax/Stern 1960, 4-7, 12-47; Ullman 1961.

78 Schreiben Arthur Israel (München) an Felix Epstein (Hamburg) vom 15.6.1967. Staatsarchiv Hamburg Bestand 611-11, Sig. 38 (2). 


\section{Die Nachkriegszeit}

Das Kriegsende gab Arthur Israel die Chance, an alte Verbindungen wieder anzuknüpfen. Er nahm wieder Kontakt zu ehemaligen Kollegen in Hamburg und Berlin und zu Bier auf. Der 85. Geburtstag August Biers stand an. Aus diesem Anlass fand am 23. November 1946 eine Feier in der Berliner Charité statt, bei der ein «russischer Professor» ${ }^{79}$ nach Aussage Biers eine «glänzende» Rede hielt, aber auch Sauerbruch und von Bramann sprachen. Bier, dem gesundheitliche Beeinträchtigungen zu schaffen machten, blieb der Feier fern, empfing aber am Tag darauf Gäste in Sauen, u.a. Domrich. Post aus dem Ausland erhielt Bier von den ehemaligen Mitarbeitern der Klinik Hans Mannheim und Alfred Horwitz (1884-?), die wie Israel das Land hatten verlassen müssen.

Auch Arthur Israel schrieb ihm aus New York. Bier antwortete Israel am 10. Januar 1947:

Dass auch Sie zum 85. Geburtstag meiner so treu gedacht haben, hat mich ganz besonders erfreut. Ich danke Ihnen herzlichst für Ihren Brief, aus dem ich ersehe, dass es Ihnen trotz aller durchgemachten Strapazen gut geht und Sie sich eine neue Existenz geschaffen haben. Die Ihnen noch fehlende befriedigende klinische Hospitaltätigkeit wird Ihnen hoffentlich auch bald zuteilwerden. - Unendlich leid tut es mir, dass Sie und Ihre Familie so überaus schwere Schicksalsschläge erlitten haben. - Mir und meiner Familie geht es den Verhältnissen entsprechend gut, wenn ich bei mir von einigen lästigen Altersbeschwerden absehe, wobei mich die stark herabgesetzte Sehkraft am meisten hindert (...)»

Nach Vermittlung von Biers Privatsekretärin (Lilli Siggelkow) ließ Israel Bier auch ein «Paket aus Amerika» zukommen, in dem alltägliche Gebrauchsgegenstände (Hosenträger und eine Krawatte) enthalten waren, wofür sich Bier im März 1947 bedankte. ${ }^{80}$

Anfang 1948 schrieb (Karl) Hermann Domrich Arthur Israel. Domrich wartete mit einer Überraschung auf, indem er Israel vorschlug, an die «alte Wirkungsstätte zurückzukehren und die Zügel dort wieder aufzunehmen, wo Sie sie 1933 aus der Hand geben mussten». Domrich betonte in dem Schreiben, dessen erste Zeilen er bereits im September 1947 verfasst hatte, seine Treue zur Klinik: «Wir haben keinen Tag die Arbeit zu unterbrechen brauchen.» ${ }^{81}$ Was er nicht erwähnte, war der eigene unsichere Status, den er als ehemaliges NSDAP-Mitglied inzwischen in der sowjetisch besetzten Zone innehatte. ${ }^{82}$

79 Es handelte sich um einen der Söhne Clara Zetkins, offensichtlich um den Chirurgen Maxim Zetkin (1883-1965), der 1945 aus der Sowjetunion nach Berlin zurückgekehrt war (Baldamus 1961, 86).

80 Nachlass.

81 Schreiben Hermann Domrichs an Arthur Israel, Anfang 1948, Nachlass.

82 Landesarchiv Berlin C Rep. 118, Nr. 1692; Dorka 1966, 439; Herrn 2010; vgl. Arndt 2009. 
Israel, der im Antwortschreiben vom März 1948 zunächst auf das «Unmaß von Bitterkeit und nicht gutzumachendes Leid», das auf ihn und seine Angehörigen «herniedergegangen» sei, verwies, war einer Rückkehr in die Ziegelstraße zugetan. ${ }^{83}$ Der Vorschlag ist offenkundig in einer FakultätsSitzung der Berliner Universität auch zur Sprache gebracht worden. Mit dem Verweis auf seine US-amerikanische Staatsbürgerschaft, die im «Russischen Sector» nicht akzeptiert werden würde, konnte dieses Ansinnen im Juni 1948 aber schnell abgeschmettert werden. Die Fakultät 〈rief〉 Israel nicht, und die Universität revidierte das Unrecht des Entzugs der Lehrbefugnis nicht. Der Gynäkologe der Charité, Walter Stoeckel (1871-1961), betonte in seinem Schreiben an Israel, dass alle, die darüber in der Fakultät debattiert hätten, Israel als «außerordentlich sympathischen Kollegen» ansehen würden, «an dem wieder viel gut zu machen» wäre «infolge der ihm zugefügten Kränkungen». ${ }^{84}$

Am 12. März 1949 starb August Bier ${ }^{85}$ In Berlin hielt Sauerbruch eine Gedenkrede auf der Sitzung der Berliner Chirurgischen Gesellschaft am 16. Mai 1949, ${ }^{86}$ in Kiel übernahm dies Karl Vogeler, der inzwischen in Rendsburg eine Privatklinik unterhielt. ${ }^{87}$ Auch Arthur Israel wurde in New York aktiv. Er schrieb sowohl Gedenkworte an Biers Sekretärin ${ }^{88}$ als auch einen Nachruf in der deutsch-jüdischen Zeitung Aufbau, die seit 1934 in New York erschien und das Vereinsblatt des New Yorker «German-Jewish Club» war. Der Aufsatz erschien am 25. März 1949 unter dem Titel «Ein großer Arzt und Lehrer» und rief damit Bier den deutsch-jüdischen Emigranten wieder in Erinnerung. Er war in Aufbau und Aussage typisch für die Festreden, die seitens seiner Schüler auf Bier gehalten wurden. Die Einführung der Lumbalanästhesie fungierte wie jedes Mal, wenn eine Lobrede auf Bier gehalten wurde, als Marker seines wissenschaftlichen Ruhms. Israel hob männliche Entschlossenheit und Weitsicht Biers hervor. ${ }^{89}$

Auf Vermittlung eines anderen deutsch-jüdischen Emigranten, des Sauerbruch-Schülers Rudolf Nissen (1896-1981), veröffentlichte Israel im Sommer eine überarbeitete und erweiterte Fassung des Aufbau-Aufsatzes im Journal des «International College of Surgeons». ${ }^{90}$ Israels Aufsatz in dem Journal

83 Schreiben Arthur Israels an Hermann Domrich, März 1948, Nachlass.

84 Schreiben Walter Stoeckels an Arthur Israel vom 18.7.1948, Nachlass.

85 Danksagungskarte der Familie vom 12. März 1949 (Michael Goerig, Hamburg: Sammlung August Bier).

86 Taubert 1992, 20-21.

87 Schreiben Karl Vogelers an Arthur Israel, Rendsburg, 2.2.1953, Nachlass.

88 Brief Lilli Siggelkows, Sauen, 22. Mai 1949, an Arthur Israel, Nachlass.

89 Israel 1949a.

90 Israel 1949b. 
war - soweit belegbar - der einzige Nachruf auf Bier in einem englischsprachigen Fachjournal.

Der alte Personenkreis der Klinik in der Ziegelstraße war für Israel imaginär positiv besetzt. Das Zugehörigkeitsgefühl zu diesem Kreis war als positiv empfundene Grundlage des eigenen Selbstverständnisses in der Emigration unangetastet geblieben.

\section{Die 1950er Jahre}

Von New York aus erwirkte Israel in den 1950er Jahren über einen Fachanwalt aus London auf Grundlage des 1953 erstmals erlassenen Bundesentschädigungsgesetzes Ausgleichszahlungen.${ }^{91}$ Seine finanzielle Situation besserte sich vor allem ab 1956 erheblich, als er vom Bundesministerium des Inneren in Bonn nachträglich zum Ordinarius ernannt wurde. Beruflich spielte das keine Rolle mehr. Praktisch kam es darin zum Tragen, dass er zeitgleich Emeritus wurde, was wesentlich bessere Altersbezüge mit sich brachte. Es war für ihn jedoch auch «ideell» wichtig. ${ }^{92}$ Ungeachtet der komplexen Problematik einer solchen «Wiedergutmachung» ${ }^{93}$ hatte sie im Fall Arthur Israels offensichtlich zur Folge, dass er eine Remigration nach Deutschland unter materiell gesicherten Verhältnissen erwägen konnte.

In dieser Zeit, als er noch in New York lebte, war es ein anderer, nach Brasilien emigrierter deutsch-jüdischer Chirurg, dessen Wirken ihn dazu veranlasste, in persönlichen Briefen seine Einstellung zur eigenen Vergangenheit kundzutun. Es handelte sich um den Urologen Paul Rosenstein (1875-1964), der Nachfolger seines Vaters im Jüdischen Krankenhaus in Berlin und zeitweise Konkurrent seines Bruders Wilhelm gewesen war. ${ }^{94}$ Rosenstein, der nicht ausschließlich mit Hochachtung über James Israel schrieb, hatte 1954 seine Memoiren unter dem Titel «Narben bleiben zurück. Die Lebenserinnerungen des großen jüdischen Chirurgen» veröffentlicht. ${ }^{95}$ Israel lehnte Rosensteins Selbstdarstellung ab und bezichtigte ihn der Eitelkeit. Vor allem lehnte er aber die vermeintliche Klagsamkeit in dessen Autobiographie ab. An den Kuratoriumsvorsitzenden des Israelitischen

91 Staatsarchiv Hamburg Bestand 314-15, Sig. F 1142, und Bestand 351-11, Sig. 6711.

92 Schreiben Arthur Israels an Felix Epstein (Hamburg) vom 23.6.1956 sowie vom 30.8.1956,

Staatsarchiv Hamburg Bestand 611-11, Sig. 38 (2).

93 Pross 1988; Goschler 2008.

94 Über Rosenstein siehe: Moll/Krischel/Rathert 2011.

95 Rosenstein 1954. 
Krankenhauses in Hamburg, Felix Epstein (1882-1982), ${ }^{96}$ schrieb Israel im Juni 1956 aus New York:

Dieses Selbstmitleid ueber die zurueckgebliebenen Narben ist nicht am Platze, wenn sechs Millionen umgebracht worden sind und ein paar Narben ihrem elenden Todesschicksal vorgezogen haetten. Bei uns allen sind Narben zurueck geblieben und bei manchen sehr viel schwerer als bei dem reichen Rosenstein, der jedes Jahr nach Europa faehrt und laecherlich wirkt er geradezu, wenn er die Welt anklagt, dass er infolge der Nazizeit an der Loesung des Krebsproblems verhindert worden sei $(\ldots)^{97}$

Unabhängig von der Polemik drückte sich darin eine Konkurrenzsituation aus, über die sich Israel erheben wollte durch eine kognitiv stringente und selbstdisziplinierende Abgrenzung zur Klage über eigene NS-Gewalterfahrungen. Die eigene emotionale Befindlichkeit blieb darin gewollt verdeckt.

\section{Die Remigration}

Bis 1959 hat Israel in New York als Arzt praktiziert. ${ }^{98}$ Im darauffolgenden Jahr kam er nach Deutschland. ${ }^{99}$ Seit dem 2. Juli 1962 ist er bei einem seiner ehemaligen Arbeitgeber, dem Mount Sinai Hospital in New York, mit der Münchener Adresse gemeldet. ${ }^{100}$ Die eigene schmerzhafte Vergangenheit erschien in abgeklärte Bahnen gelenkt, als die Feierlichkeiten zu Biers 100. Geburtstag 1961 näherrückten. Das Jahr der zementierten deutsch-deutschen Teilung führte jedoch auch bei Israel zu einer Krise.

In Berlin sollte der Gedenktag im Rahmen der Berliner Chirurgischen Gesellschaft begangen werden. Der Bau der Berliner Mauer am 13. August kam dem zuvor. Die letzte gemeinsame Sitzung der «Gesellschaft» vor ihrer Teilung infolge des Mauerbaus fand am 19. Juni 1961 statt. Im Sommer gab es, traditionsgemäß, keine Sitzungen. ${ }^{101}$ Im Herbst standen die Gedenkfeiern zu Ehren Biers an. Die Ostberliner «Gesellschaft» kam am 23. November 1961 vormittags im Hörsaal der Chirurgischen Klinik der Charité zusammen. ${ }^{102}$ Die Westberliner «Gesellschaft» traf sich am 24. November abends im Städtischen Krankenhaus Westend. Nach der offiziellen (Westberliner)

96 Über Epstein siehe: Heinsohn 2009.

97 Arthur Israel an Felix Epstein, 23.6.1956, Staatsarchiv Hamburg Bestand 611-11, Sign. $38(2)$.

98 Bloch/Israel/Schultze-Seemann 1983, 90.

99 Schreiben Arthur Israels (München) an Felix Epstein (Hamburg) vom 10.2.1961, Staatsarchiv Hamburg Bestand 611-11, Sig. 38 (2).

100 Medical Staff Office: Personalkartei, Karte zu «Israel, Arthur, MD.», Archives of the Mount Sinai Hospital New York.

101 Mündliche Mitteilung Gert Specht (Berlin), 10.4.2012.

102 Taubert 1992, 24. 
Veranstaltung kamen ehemalige Mitglieder der Klinik in der Ziegelstraße mit Angehörigen der Familie Bier zu einem Festessen im Westberliner «Hotel am Zoo» (Berlin-Charlottenburg) zusammen. Aus Rendsburg war Karl Vogeler zugegen, der unter dem Vorsitz Constantin von Bramanns ${ }^{103}$ als Hauptredner der Veranstaltung im Westend-Krankenhaus über «August Biers Lebenskampf» sprach. ${ }^{104}$

Israel hatte Monate zuvor den Deutschen Chirurgenkongress in München besucht und dort mit zwei ehemaligen Bier-Schülern und einem Angehörigen gesprochen. Seinem in Amsterdam lebenden besten Freund Albert Salomon (1883-1976) ${ }^{105}$ gegenüber, der selbst als Chirurg unter Bier habilitiert worden war und vor den Nazis hatte fliehen müssen, bekannte er:

Sonst aber ist man dort ein Fremder geworden. In dem dicht gefüllten Saal des Deutschen Museums sah ich kaum ein bekanntes Gesicht. Und diese Wenigen aus der Ziegelstraßenzeit sind in solchen Tagen mit den Verhandlungen, mit sich selber, mit Freunden und ihren Familien beschäftigt (,) um, so liebenswürdig sie auch sind, viel Zeit für ein Zusammensein aufzubringen. ${ }^{106}$

Er zögerte zunächst, an den Veranstaltungen in Berlin teilzunehmen, um einer möglichen Enttäuschung zu entgehen. ${ }^{107}$ Er tat es aber doch, und insbesondere das Festessen muss für ihn emotional berührend gewesen sein wie ein Familientreffen, das nach langer Zeit wieder stattfinden konnte. ${ }^{108}$ Er hielt eine ganz oder in Teilen improvisierte Rede, die alle Ingredienzien des emphatischen Lobes auf Bier enthalten haben muss. ${ }^{109}$ Sie wurde auf Tonband mitgeschnitten, ${ }^{110}$ der Mitschnitt ist jedoch nicht mehr überliefert. Vogelers Rede ist noch im Dezember desselben Jahres den üblichen Gepflogenheiten gemäß in der Fachpresse publiziert worden. ${ }^{111}$ Israel wurde

103 Wortlaut der Ansprache siehe v. Bramann 1962.

104 Vogeler 1962.

105 Schriftwechsel Arthur Israel / Albert Salomon, Nachlass.; Schwoch 2009, 761-762; FischerDefoy 1992.

106 Arthur Israel an Albert Salomon vom 19.5.1961, Nachlass.

107 v. Bramann entgegnete Israel am 16.11.1961 auf ein Schreiben, das nicht erhalten ist: «Für Ihren Brief vom 14.11. herzlichen Dank. Ich möchte Ihnen nochmals sagen, dass uns gerade an Ihrer Teilnahme an dem Abend des 24. Nov. ganz besonders viel gelegen ist. Ich respektiere Ihre Bedenken, doch bin ich davon überzeugt, dass nicht nur die Bier-Schüler, sondern auch die zahlreichen Mitarbeiterinnen und Mitarbeiter der Bier'schen Klinik, welche ihr Erscheinen zugesagt haben, sich ganz besonders über Ihr Erscheinen freuen würden. Es trifft nicht zu, dass nur die Damen Zimmermann Sie auch heute noch verehren, viele Andere der geladenen Gäste würden sich über Ihre Teilnahme freuen (...)» (Schreiben Constantin von Bramanns an Arthur Israel vom 16.11.1961, Nachlass).

108 Schreiben Arthur Israels an Constantin von Bramann vom 20.12.1961, Nachlass.

109 Domrich 1963, 495.

110 Vogeler 1961; Domrich 1963; Nachlass.

111 Vogeler 1962; vgl. Vogeler 1961. 
ebenfalls sogleich aufgefordert, die Rede zur Publikation in der Zeitschrift Berliner Medizin einzureichen. ${ }^{112}$

Genau das konnte er jedoch nicht. «Leider hatte ich meine Ansprache», schrieb er im Dezember 1961 ausflüchtend an v. Bramann, «nur skizziert und das Meiste aus dem Augenblick heraus frei geformt, dabei manches nicht zu meiner Zufriedenheit klar genug ausgeführt, anderes, was als Abschluss wichtig gewesen wäre, fortgelassen, um die Hörer nicht zu ermüden.» Eine schriftliche Fassung sei ungleich «verantwortungsvoller» als eine mündliche Version, und gesundheitliche Beschwerden würden ihn belasten. ${ }^{113}$

So ging es weiter. Im Juni 1963 verwies Israel v. Bramann gegenüber darauf, dass die «Geistesart» Biers eine «tiefere Analyse» erfordere, als er sie bei der freien Rede habe geben können. Eine «besser formulierte Fassung» könne er aber noch nicht vorlegen. ${ }^{114}$ Der Kardiologe Ernst Wollheim (1900-1981), ${ }^{115}$ der einst in Berlin tätig gewesen war und mit Israel die Erfahrung der Emigration teilte, forderte Israel in einem Zeitschriftenbeitrag aus Anlass von dessen 80. Geburtstag auf, die vergangenen Zeiten noch einmal schriftlich aufleben zu lassen:

Mein besonderer Geburtstagswunsch wäre, dass er [Israel] diese Gnade [geistiger Frische und Gesundheit] ausnutzt und seine Erinnerungen an die große medizinische Vergangenheit Berlins bis zum Ausbruch des 3. Reiches niederschreibt. Kein anderer wäre so geeignet wie er, jenen wichtigen Zeitabschnitt uns und denen, die nach uns kommen, im Gedächtnis zu bewahren. ${ }^{116}$

(Karl) Hermann Domrich war inzwischen Nachfolger v. Bramanns als Vorsitzender der Westberliner «Gesellschaft». Im Februar 1964 teilte Israel Domrich mit, dass er noch nichts Druckreifes vorlegen könne. ${ }^{117}$ Seinem Freund Albert Salomon gestand Arthur Israel im Juli 1964 seine Zweifel in selbstanklagender Verzagtheit:

(...) Wie schwer ist es doch, wenn man es wirklich mit letzter wissenschaftlicher psychologischer und geistesgeschichtlicher Präzision fassen will, eine Persönlichkeit wie die von Bier in ihrem tiefsten Wesen in Worten lebendig zu machen. Das übliche Festartikel Geschwätz mit all den grauenhaften Schlagworten von Ganzheit und der Beschreibung abgestandener, verfehlter Arbeiten des Mannes, ist doch unerträglich. Und was gar nicht leicht ist, das ist der Einbau eines Menschen in sein Zeitalter, in die Entwicklung der naturwissenschaftlichen, medizinischen, biologischen Anschauungen, nicht zu vergessen, auch die philosophischen. Und wenn man sich mit solchem Zeug eingehend beschäftigt, dann sieht man erst, wie wenig man wirklich Bescheid weiß, wie an allen Ecken und Enden sich Wissenslücken auftun und man Buch um Buch studieren muss, um mit neuerlangtem Wissen die Lücken zu stopfen.

112 Schreiben Constantin von Bramanns an Arthur Israel vom 16.12.1961, Nachlass.

113 Schreiben Arthur Israel an Constantin von Bramann, 20.12.1961, Nachlass.

114 Arthur Israel an Constantin von Bramann, 29.06.1963, Nachlass.

115 Schwoch 2009, 915-918; Festkomitee 1960, 95.

116 Wollheim 1963a, 1818; vgl. Wollheim 1963b.

117 Arthur Israel an Hermann Domrich, 29.2.1964, Nachlass. 
Diese Vorstellungen haben bei mir eine Art Lethargie erzeugt. Ich habe wohl viel Wissen eingeatmet, aber nichts Rechtes ausgeatmet (...) $)^{118}$

Salomons Reaktion wird ihm nicht viel weitergeholfen haben. Er entgegnete Israel wenig später knapp, er solle sich nicht so quälen mit seinem BierArtikel, das ganze «Genie» könne er nicht erfassen $[\ldots]^{119}$

\section{Zweifel an der Klinik in der Ziegelstraße}

In München angekommen, versuchte Arthur Israel im intellektuellen Umfeld der Stadt Erlebtes und Erlittenes in Worte zu fassen. Er sah sich um, las, hörte Vorträge an der Universität und im Rundfunk. Aus einer englischsprachigen Illustrierten riss er einen Aufsatz heraus, der eine Zusammenfassung eines ausführlichen Beitrags im deutschen Nachrichtenmagazin Spiegel enthielt. Der Beitrag im Spiegel über die Lage der Juden in Deutschland, erschienen im Juli 1963, begann mit den Worten: «Sie wohnen in Deutschland und mißtrauen den Deutschen. Sie bekennen sich als Juden und müssen sich von Juden in der übrigen Welt behandeln lassen wie verlorene Söhne.» ${ }^{120}$ Der englischsprachige Beitrag war übertitelt mit «Jews in Germany now - Psychological Tensions». ${ }^{121}$

Israel hat, gemäß dem vorliegenden Berliner Nachlass, damals nur wenig Zeitungsausschnitte gesammelt. Einen Artikel des österreichischen Arztes, Zoologen und vergleichenden Verhaltensforschers Konrad Lorenz (19031989), den dieser anlässlich des Erscheinens seines Buches Das sogenannte Böse ${ }^{122}$ geschrieben hat, hat Israel jedoch im August 1964 aufgehoben. Lorenz, dessen ethisches Verständnis von Überlegungen zur Evolution von Mensch und Tier geprägt war, schrieb damals:

(...) Wir sind das Höchste, was die großen Konstrukteure des Artenwandels auf Erden bisher erreicht haben, wir sind ihr <letzter Schrei〉, aber ganz sicher nicht ihr letztes Wort. Für den Naturforscher sind alle Absolutsetzungen - selbst solche auf erkenntnistheoretischem Gebiet - verboten, sind Sünde gegen den heiligen Geist des Alles fließt, der großen Erkenntnis des Herakleithos, daß nichts ist, sondern alles in ewigem Werden dahinströmt. $(\ldots)^{123}$

118 Schreiben Arthur Israel an Albert Salomon, 9.7.1964, Nachlass.

119 Schreiben Albert Salomon an Arthur Israel, 28.7.1964, Nachlass.

120 Heimstätte 1963, 24.

121 Jews in Germany Now. Psychological Tensions. Zeitschrift nicht eruiert, naheliegenderweise aus dem Jahr 1963, S. 58, Nachlass.

122 Lorenz 1974.

123 Lorenz 1964. 
Israel wird von Lorenz' intensiven Verstrickungen in das nationalsozialistische Regime ${ }^{124}$ wahrscheinlich keine Kenntnis gehabt haben, als er 1962 erstmals mit ihm Kontakt aufgenommen hatte. Rundfunkvorträge zur Frage des «Bösen» und der Aggression, die Lorenz gehalten hatte, hatten ihn dazu motiviert. ${ }^{125}$ Lorenz' Reminiszenz an Heraklit wird ihn an seinen Lehrer August Bier gemahnt haben, der Heraklit stets im Munde geführt hatte.

Lorenz war damals Zoologie-Professor in München und Direktor des Max-Planck-Instituts für Verhaltensphysiologie in Seewiesen am Eßsee. Lorenz erwähnte in dem Vortrag, auf den Israel sich bezog, seinen einstmaligen Mentor, den Zoologen Oskar Heinroth (1871-1945). Bier hatte diesen nach Aussage Israels dereinst in Fragen der Zoologie um Rat gefragt, was ihn, Israel, jetzt veranlasste, sich mit demselben Ansinnen an Lorenz zu wenden. In einem Schreiben vom 25. Februar 1962 skizzierte Israel Biers Persönlichkeit und erwähnte, dass «Hippokrates und der Philosoph Heraklit» seine «Abgötter» gewesen seien. In seinem Buch Die Seele habe Bier schon ausgeführt, dass das Gute und das Böse zusammen zur Harmonie führen würden. Ein Heraklitiker würde in allem einen Logos, also einen Sinn, sehen. Israel berichtete aber auch von den Schwierigkeiten, die ihm die Bier'sche Weltanschauung inzwischen bereiten würde:

In den Feiern und Festartikeln zum Gedenken an seinen 100. Geburtstag im letzten November wurde stets an Biers teleologischen Standpunkt gedacht, den er einst in schwungvollen Worten zum Ausdruck gebracht hat. - Und ich glaube auch, dass die Teleologie - mit $\mathrm{Ma} ß$ - eine gute heuristische Methode gewesen ist. Ich selbst sprach an jenem Festtage ein paar Worte und wurde aufgefordert, meine Gedanken schriftlich zu fassen.

Aber je mehr ich mich mit dem Thema befasste, umso mehr hatte ich Hemmungen, umso mehr stießen, bei all meiner Verehrung für die Person und für die Gabe seiner Schau der Phänomene Zwe[i]fel auf an der Zukunftsträchtigkeit seiner Auffassungen von der Anwendbarkeit der antiken naturphilosophischen Vorstellungen auf die naturwissenschaftlichen und medizinischen Probleme der Gegenwart.

Wo Israel vorsichtig anfragte, kam Lorenz, der mit radikalen Urteilen kein Problem hatte, unumwunden zur Sache. Aus seiner Geringschätzung für Bier machte er in seinem polemischen Antwortschreiben vom 22. März 1962 keinen Hehl:

Ihrer Meinung über August Bier kann ich mich leider nicht anschließen. Nach meiner Meinung ist die 〈Bier-Seele〉, wie dieses schreckliche, Gott sei Dank längst vergessene Büchelchen bei Heinroth und mir hieß, ein schlagendes Beispiel dafür, wie eine schlechte Teleologie alle Vorstellungen vom stammesgeschichtlichen Werden und Anpassung vollkommen durcheinander bringt. Ich glaube, dass die Überheblichkeit und Eitelkeit, wie sie chirurgischen Klinikchefs so häufig eignet, dazu notwendig ist, so unkritischen Unsinn zusammen zu schreiben, wie Bier es getan hat (...)

124 Vgl. Föger/Taschwer 2001.

125 Schriftwechsel Arthur Israel / Konrad Lorenz, Nachlass; vgl. Deich 1964. 
Das war starker Tobak. «Arthur Israel, New York, z.Z. München» wagte sich nun aus der Reserve und schlug am 2. Mai 1962 einen für ihn völlig untypischen schroffen Ton an:

(...) Vor allem aber bestätigte Ihre ablehnende Kritik von Bier's metaphysischen Versuchen die Fragwürdigkeit einer Denkweise, die mir immer fremd gewesen war. Bier's überwuchernde naturphilosophischen Neigungen entfernten ihn in seinem späteren Lebensabschnitt immer mehr von der wissenschaftlichen Methodik. Sein heraklitisches> Weltbild war kindlich primitiv und seine Verliebtheit in die Antike, besonders in die Hippokratische Welt schien mir oft eine wissenschaftsfeindliche Flucht in das Archaische zu sein. Das Merkwürdige ist nur, das[s] trotz allen diesen berechtigten Einwänden Bier in der Klinik bei kritischen und unkritischen Mitarbeitern die größte Hochachtung genoss. Ist doch die Persönlichkeit mehr als die Summe der einzelnen Vorzüge und selbst Widersprüche und Irrungen können ihr eine gewisse Farbigkeit geben. ${ }^{126}$

Ein Rundfunkvortrag - über «Humanismus und Christentum» - war es im Jahr 1964 wiederum, der Israel veranlasste, sich an einen anderen prominenten Denker der Nachkriegsjahrzehnte zu wenden: den Münchener Philosophen Helmut Kuhn (1899-1991). Kuhn hatte - als «Halbjude» - ebenfalls nach 1933, im Jahr 1937, seine Zeit als Privatdozent an der Berliner Universität beenden müssen und war in die USA emigriert. Er entwickelte in den USA und - nach dem Krieg - in Deutschland nicht nur seine an der griechischen Philosophie geschulte Metaphysik weiter. Er wandte sich auch dem Katholizismus zu und setzte sich seit seiner Zeit in den USA in Schrift und Tat gegen den Nationalsozialismus und für eine Demokratie nach US-amerikanischem Vorbild ein. ${ }^{127}$

Als Israel 1965 einen Vortrag Kuhns über das rechtsradikale geistige Klima bei Professoren und Studenten nach dem Ersten Weltkrieg gehört hatte, berichtete er diesem am 22. November von seinen eigenen Erfahrungen:

Als Zeuge jener Zeit - als Assistent, Privatdozent und a.o. Professor in der chirurgischen Universitätsklinik - kann ich nur allzu gut jene geistigen Strömungen dieser Epoche beurteilen, den allgemein herrschenden anti-demokratischen Widerwillen gegen die Republik vom Chef bis zum jüngsten Mitarbeiter des Instituts. Man starrte nostalgisch auf den militärischen Glanz des Hohenzollern Reiches zurück. Noch heute fühle ich die Erschütterung, als ich am Tage nach der Ermordung von Rathenau am Nachmittag die Klinik betrat. Professor Bier und die Assistenten waren alle im Operationssaal versammelt. Die Atmosphäre einer heiteren Erregtheit lag in der Luft, ja ich hatte das Gefühl, man habe sich zur Feier eines Siegesfestes eingefunden. Ich war tief betroffen. Aber wenn ich auch nur ein gutes Wort über den Ermordeten fallen ließ, so begegnete mir kühles Schweigen. Ich konnte mich schließlich nicht enthalten, von der Untat des Rechts-Gesindels zu sprechen. Am nächsten Tag bat mich der Oberarzt in einer Aussprache, dass meine Bemerkung allgemeines Befremden erregt habe. Ich antwortete, es gäbe Rechts- und Links-Gesindel, die eine Entartung

126 Schriftwechsel Arthur Israel / Konrad Lorenz, Nachlass.

127 Seine Tochter, die feministische Historikerin Annette Kuhn (geb. 1934), hat sich später kritisch mit seiner Grundeinstellung, die sie als autoritär charakterisierte, auseinandergesetzt (Kuhn 2003). 
der rechten und linken Parteien seien. Die Parteinahme für die Mörder war offensichtlich. Dies war die Stellungnahme von Ärzten, die nach den Priestern der nächste Stand hätten sein sollen [,] um sich der Inhumanität dieses Verbrechens entgegenzustellen. Dies geschah 10 Jahre bevor der Nationalsozialismus zur Herrschaft kam - aber jener Mord war schon ein Fanal. ${ }^{128}$

Weitere kritische Äußerungen Israels sind nicht überliefert. Es ist - soweit nachweisbar - auch zu keiner Publikation Arthur Israels über August Bier mehr gekommen.

Gesundheitliche Beeinträchtigungen machten Israel nun immer wieder zu schaffen. Im Jahr 1961 hatte er bereits einen Herzinfarkt bei Diabetes mellitus hinter sich und litt unter einer Herzkrankheit durch chronischen Bluthochdruck. ${ }^{129}$ Zwei Tage nach seinem 86. Geburtstag, am 27. April 1969, ist er gestorben. ${ }^{130}$ Die Kritik an Bier und dem Kreis der Klinik in der Ziegelstraße blieb unvollständig. Der Bruch, der in dieser beginnenden Kritik lag, erfordert eine hypothetische Interpretation.

\section{Synopse}

Das stereotype Bild des Chirurgen bezeichnet einen «action-oriented male hero», der einsam waghalsige Entscheidungen trifft und riskante Schritte wagt. Die Chirurgie wird als Drama erlebt. Der Chirurg ist typischerweise männlich. Obzwar es eine «general surgical culture» gibt, differieren die persönlichen Stile von Chirurgen substantiell. Die «surgical culture» perpetuiert durch die Rekrutierung und lange Ausbildung angehender Chirurgen, die vom Bild des etablierten Chirurgen, seinem Prestige, seinem Einfluss und dem «heroic mystique», das ihn umgibt, angezogen werden. Überzeugungen, Werte, Vorstellungen werden innerhalb der «surgical culture» tendenziell geteilt. So hat es der amerikanische Anthropologe Pearl Katz 1999, vorrangig mit Blick auf die USA, herausgearbeitet. ${ }^{131}$ August Bier entsprach in den Augen derer, die ihm folgten, und vieler anderer Zeitgenossen mustergültig dem Bild des chirurgischen «Helden», dessen Ruhm markiert war durch die

128 Schriftwechsel Arthur Israel / Helmut Kuhn, Nachlass. - Der liberale jüdische Unternehmer und Reichsaußenminister Walther Rathenau (1867-1922) ist am 24.6.1922 von einem rechtsradikalen Geheimbund ermordet worden. Rathenau war gut bekannt gewesen mit der Familie Israel (Bloch/Israel/Schultze-Seemann 1983, 89-90). Bei dem genannten 1. Oberarzt müsste es sich um Rudolf Klapp (1873-1949) gehandelt haben. Zu Klapp siehe: Blau 2007.

129 Schreiben Max Hösel (Ulm) an Arthur Israel, 22.4.1961, Nachlass.

130 Medical Staff Office: Personalkartei, Karte zu «Israel, Arthur, MD.», Archives of the Mount Sinai Hospital New York.

131 Katz 1999. 
im «heroischen» Selbstversuch begründete Einführung der Spinalanästhesie für chirurgische Zwecke.

Für Arthur Israel verkörperte August Bier das Ideal des Chirurgen. Die Divergenz der Charaktere trübte das persönliche Verhältnis, das nach überkommenem Muster ein Lehrer-Schüler-Verhältnis sein musste, nicht. Bier war die prägende Persönlichkeit in Israels Leben. Zweifel an der Person des Lehrers waren nicht grundsätzlicher Art. Die Person blieb, da grundverschieden, in letzter Konsequenz unergründlich. Erst nach dessen Tod konnte Israel im halböffentlichen Schriftwechsel Kritik äußern, indem er dessen späte Naturphilosophie als unwissenschaftlich oder naiv geißelte. Persönliche Kritik an Bier war Israel auch 1947 noch nicht möglich, als Bier das «Schicksal» apostrophierte, um Israels Leidensweg zu kennzeichnen. Der in seiner Zeit als sprachgewaltig geltende Bier hatte keine Worte gefunden für die Ursache und die Verursacher der Misere, in der sich Arthur Israel und andere deutsch-jüdische Ärzte befunden hatten. Das Unglück war von außen gekommen und hatte anscheinend vormals ungetrübte Verhältnisse zerrüttet.

Solange Israel das «gute Gewissen» der Klinik war, diese nach Biers Ausscheiden weiterführte und es auch nach der Vertreibung als Pflicht ansah, Biers Ruf und den seiner Klinik in Ehren zu halten, tat er dies kund in Festreden. Die Irritation, die Israel 1923 angesichts der Reaktion der ärztlichen Kollegen in der Ziegelstraße auf die Ermordung Rathenaus empfand, scheint nicht mehr als eine Momentaufnahme gewesen zu sein. Reflektiert hat er dies anscheinend erst vierzig Jahre später.

Die Festrede auf Bier bestärkte den Zusammenhalt der Klinik Biers im Sinne einer Zugehörigkeit zu einem Kreis Auserwählter, der imaginierten «Schule Biers». Durch die Festrede wurde Zugehörigkeit dokumentiert und tradiert. Dies korrespondierte im Fall Israels mit einer aus dem Elternhaus übernommenen konservativen Weltsicht. Als Bier 1949 gestorben war, hielt es niemand im Ausland für angebracht, einen Nachruf zu verfassen - außer einigen aus Europa stammenden jüdischen Ärzten, von denen es wiederum Israel zukam, die lobenden Worte zu verfassen. Israel war geradezu der Spezialist für Lobreden auf Bier und die Klinik in der Ziegelstraße.

Die nationalsozialistische Gewaltherrschaft zerstörte die alte Ordnung. Der passiv erlebte Bruch der Jahre 1933 und 1940 hob sich von der vor-vergangenen Zeit ab wie das gewaltsame Ende einer rundherum positiv bewerteten, intakten Welt im prägenden Mikrokosmos der Ziegelstraße oder nach dessen Muster (in Hamburg). Israels Exil war eine emotional dürftige und materiell bescheidene Zeit, die dadurch bestimmt war, dass das Überleben zu sichern war. Die ideellen Grundfesten blieben dieselben. 
Als das faschistische Regime in Deutschland abgedankt hatte, erlaubte sich Israel allem Anschein nach im kommunikativen Umgang weiterhin keine «lauten» Emotionen: keinen Hass, keine Abscheu, keine Wut. In den 1950er Jahren erklärte er es ausdrücklich als unangemessen, seine «Narben» zu zeigen. Gleichzeitig hat er, soweit belegbar, nie persönliche Verurteilungen ehemaligen Kollegen aus der Ziegelstraße gegenüber ausgesprochen, obzwar auch Schüler Biers (z.B. Vogeler oder Domrich) für den Betrieb der Klinik unter den Gewaltherrschern verantwortlich gezeichnet hatten.

Eine Karriereoption an alter Wirkungsstätte in Ost-Berlin wurde $1948 \mathrm{im}$ Keim erstickt. Die «Wiedergutmachung» im Westen Deutschlands bescherte Israel einen ideellen (der Titel als Ordinarius) und einen materiellen $\mathrm{Zu}$ gewinn, der 1960 die Remigration erlaubte. Nach eigenen Aussagen begann Israel nun, sich emotional wieder zu öffnen. Die emotionale Öffnung galt neben Hamburger Bekannten in erster Linie den alten personellen Zusammenhängen des Kreises der ärztlichen und anderen Mitarbeiter der Klinik in der Ziegelstraße. Sie schien sich positiv zu erfüllen, als 1961 - unter dem Signum August Biers - ein gemeinschaftliches persönliches Treffen in WestBerlin realisiert werden konnte. Erneut wurde die vermeintliche Tradition in Festreden revitalisiert. Der Emphase des Moments widersetzte sich aber die Ambivalenz, die Israel nun umtrieb, wenn er die alte Welt der Ziegelstraße, die er stets in Ehren gehalten hatte, gewärtigte.

Im Gedankenaustausch mit Münchner Gelehrten in den 1960er Jahren keimten Zweifel an der eigenen Weltsicht auf, die vom Ziegelstraßen-Kosmos bestimmt war. Verwirrung und Verunsicherung, vielleicht auch Wut auf Mitläufer und Täter kennzeichneten die Ambivalenz, die den aktiven Bruch mit der bis dahin eingenommenen Perspektive anzeigte. Dies nicht eindeutig wenden zu können, kreidete sich Israel selbst an, wenn er verzagt feststellte, die Uneindeutigkeit repräsentiere die eigene kognitive Unfähigkeit, die komplexen Zusammenhänge zu erfassen.

Die Auswertung der Quellen, die Auskunft über das Leben Arthur Israels zu geben erlauben, belegt diesen Bruch, beginnend im Jahr 1961. Hypothetisch könnte er gedeutet werden als der Verlust der Überzeugung, den positiven Kern der eigenen Lebensgeschichte als Teil der überragenden Geschichte der Klinik in der Ziegelstraße zu deuten. Die «Ziegelstraße» hatte - vor den Nazis - für Israel den Nimbus der von Bier bestimmten «Heldengeschichte» gehabt. Das Pendant der «Heldengeschichte» war die Festrede als Eloge. Die Festrede im herkömmlichen Stil wurde unmöglich, als sich die «Heldengeschichte» selbst erledigte. Sie war nicht lediglich von vermeintlich von außen gekommenen Nationalsozialisten pervertiert worden, sondern war in sich falsch. Mit dem Ende der Eloge auf Bier konnte Israel 
kein Loblied mehr auf die große medizinische Vergangenheit Berlins anstimmen. Wenn die medizinische Vergangenheit Berlins vor den Nationalsozialisten nicht einfach nur großartig gewesen war, was war sie dann? Auf diese Frage scheint Arthur Israel keine Antworten mehr gefunden zu haben.

Eine solche alternative Lesart des Lebensweges eines deutsch-jüdischen Arztes in und ausgehend von der Zeit des «Dritten Reiches» verweist die Brüche im Leben des Betroffenen nicht nur auf die Zeit ab 1933, sondern prinzipiell auch auf die komplexen Zusammenhänge der Zeit vor 1933.

\section{Bibliographie}

Nachlass Arthur Israel, Institut für Geschichte der Medizin der Charité Berlin, S $910-$ KS 18

Arndt, Melanie, Gesundheitspolitik im geteilten Berlin 1948 bis 1961 (Köln, Weimar, Wien 2009)

Baader, Gerhard, «Politisch motivierte Emigration deutscher Ärzte», Berichte zur Wissenschaftsgeschichte 7 (1984) 67-84

Baldamus, Margarethe, «August Bier zum 100. Geburtstag», Geschichtsblätter für Waldeck 53 (1961) 1-101

Berberich, Joseph/Henry Lax/Rudolf Stern, Rudolf Virchow Medical Society in the City of New York, N. Y. Jubilee Volume, 100th Anniversary / Festschrift zur 100-JahrFeier November 7, 1860-1960 (Basel, New York 1960)

Bier, August, Über die Berechtigung des teleologischen Denkens in der praktischen Medizin. Festrede gehalten am Stiftungstage der Kaiser Wilhelms-Akademie für das militärärztliche Bildungswesen, 2. Dezember 1909 (Berlin 1910)

Bier, August, «Ueber medizinische Betrachtungsweisen, insbesondere über die mechanische und über die telelogische», Münchener Medizinische Wochenschrift 69 (1922) 845-849

Bier, August, Die Seele (München 1939, 4. Aufl. 1939, 6. Aufl. 1940, 8. Aufl. 1941, 10. Aufl. 1944,11. Aufl. 1951 (62.-65. Tausend)

Bier, August, Das Leben (München 1951)

Blau, Evelyne, Rudolf Klapp. Marburger Chirurg und Orthopäde im Dritten Reich (Marburg 2007)

Bloch, Peter/James Israel/Fritz Schultze-Seemann, James Israel 1848-1926 (hrsg. von Rolf Winau) (Wiesbaden 1983)

(von Bramann, Constantin) «Festsitzung der Berliner Chirurgischen Gesellschaft aus Anlass des 100. Geburtstages von August Bier am 24. Nov. 1961», Berliner Medizin 13 (1962) 351-352

Brandmann, Hans, «Als Assistenzarzt 1936-1938 am I. K.», in: Ina Lorenz, «Die dunklen und schweren Jahre (1933-1934)», in: Israelitisches Krankenhaus Hamburg (Hrsg.), 150 Jahre Israelitisches Krankenhaus in Hamburg (Hamburg 1997) 65-85

Brandt, Karl, «Die Schließung der Bierschen Klinik», in: Paul Diepgen/Paul Rostock, Das Universitätsklinikum in Berlin. Seine Ärzte und seine wissenschaftliche Leistung 1810-1933 (Leipzig 1939) 183-188

Brenner, Michael, Kleine jüdische Geschichte (München 2008)

348 Gesnerus $70(2013)$ 
Damskis, Linda Lucia, Zerrissene Biografien. Jüdische Ärzte zwischen nationalsozialistischer Verfolgung, Emigration und Wiedergutmachung (München 2009)

Deich, Friedrich, «Aggression und Moral», Süddeutsche Zeitung 7./8. März 1964

Domrich, Hermann, «40 Jahre Lumbalanästhesie», Archiv für klinische Chirurgie 197 (1940) 792-819

Domrich, Hermann, «Prof. Dr. Arthur Israel 80 Jahre alt», Berliner Medizin 14 (1963) 495-496

Doms, Misia Sophia, «August Biers Aufsatz <Wie sollen wir uns zu der Homöopathie stellen?> (1925) und die nachfolgende Diskussion um die Homöopathie in der deutschen Ärzteschaft», Medizin, Gesellschaft und Geschichte 23 (2004) 243-282

Dorka, Günther, «Professor Hermann Domrich zum 65. Geburtstag», Forschung, Praxis, Fortbildung 17 (1966) 439

Etzemüller, Thomas, «Die Form 〈Biographie〉 als Modus der Geschichtsschreibung. Überlegungen zum Thema Biographie und Nationalsozialismus», in: Karl Heinrich Pohl/Michael Ruck (Hrsg.), Regionen im Nationalsozialismus (Bielefeld 2003) 71-90

Fangerau, Heiner/Matthis Krischel, «Der Wert des Lebens und das Schweigen der Opfer: Zum Umgang mit den Opfern nationalsozialistischer Verfolgung in der Medizinhistoriographie», in: Stefanie Westermann/Richard Kühl/Tim Ohnhäuser (Hrsg.), NS-«Euthanasie» und Erinnerung. Vergangenheitsaufarbeitung - Gedenkformen - Betroffenenperspektive (Berlin 2011) 19-28

Festkomitee des Rates der Medizinischen Fakultät zur Vorbereitung der 250-JahrFeier der Charité, 250 Jahre Charité (Berlin 1960)

Fischer, Stefanie, «Tagungsbericht <Jüdische Perspektiven auf die Jahre der forcierten Auswanderung> bis zur Ghettoisierung und Deportation der Juden aus dem Deutschen Reich (1938/39-1941) 17.5.2009-19.5.2009, Hamburg», H-Soz-u-Kult 10.8.2009

Fischer-Defoy, Christine, Paula Lindberg-Salomon - <Mein C'est la vie-Leben $\rangle$ in einer bewegten Zeit. Der Lebensweg der jüdischen Künstlerin (Berlin 1992)

Föger, Benedikt/Klaus Taschwer, Die andere Seite des Spiegels. Konrad Lorenz und der Nationalsozialismus (Wien 2001)

Goerig, Günter [fehlerhafte Angabe, tatsächlich: Michael]/Jochen Schulte am Esch, «Zur Erinnerung an August Bier (1861-1949)», AINS 34 (1999) 463-474

Goerig, Michael/Jochen Schulte am Esch, Die Entwicklung des Narkosewesens in Deutschland von 1890-1930 (Lübeck 2012)

Goschler, Constantin, Schuld und Schulden. Die Politik der Wiedergutmachung für NS-Verfolgte seit 1945 (Göttingen 2005, 22008)

Harig, Georg, «August Bier und die Geschichte der Medizin», Zentralblatt für Chirurgie 111 (1986) 1404-1410

Hartung-von Doetinchem, Dagmar/Rolf Winau, Zerstörte Fortschritte. Das Jüdische Krankenhaus in Berlin. 1756-1861-1914-1989 (Berlin 1989)

Hauser, Dorothea, «Zwischen Gehen und Bleiben. Das Sekretariat Warburg und sein Netzwerk des Vertrauens 1938-1941», in: Susanne Heim/Beate Meyer/Francis R. Nicosia (Hrsg.), «Wer bleibt, opfert seine Jahre, vielleicht sein Leben». Deutsche Juden 1938-1941 (Göttingen 2010) 115-133

[Heimstätte] «Heimstätte auf verfluchter Erde? SPIEGEL-Report über Juden in Deutschland», Spiegel Nr. 31/1963 (31.7.1963) 24-38 
Heinsohn, Kirsten, «Felix Epstein (1882 bis 1982) - ein Hamburger Bürger», in: Joist Grolle/Matthias Schmoock (Hrsg.), Spätes Gedenken. Ein Geschichtsverein erinnert sich seiner ausgeschlossenen jüdischen Mitarbeiter (Hamburg 2009) 37-64

Henschen, Carl, «Reflexionen zu August Bier’s Buch «Die Seele»», Medizinische Welt 20 (1951) 756-758

Herrn, Rainer, «Die Charité als Politobjekt: Politisches Handeln», in: ders./Laura Hottenrott (Hrsg.), Die Charité zwischen Ost und West 1945-1992. Zeitzeugen erinnern sich (Berlin 2010) 110-111

Israel, Arthur, Klinische Beobachtungen aus der medizinischen Klinik in Straßburg über das Symptom der Hypertension (Med. Diss. Straßburg 1907; Leipzig 1907)

Israel, Arthur, «Ein großer Arzt und Lehrer. Zum Tode des Chirurgen August Bier», Aufbau 25.3.1949, 7-8 (= Israel 1949a)

Israel, Arthur, «In Memoriam: August Bier, M.D. 1862-1949» Journal of the International College of Surgeons 12 (1949) 595-596 (= Israel 1949b)

Israel, James, Meine Reise zum Sultan. 10. Juni bis 3. August 1915. Tagebuchblätter des Chirurgen und Urologen. Mit einer biographischen Skizze von Rolf Winau (Teetz 2006)

Israel, James/Wilhelm Israel, Chirurgie der Niere und des Harnleiters. Ein Lehrbuch (Leipzig 1925)

Jacob, Ruth, «Zwölf exemplarische Biografien - eine Einführung», in: Ruth Jacob/ Ruth Federspiel (Hrsg.), Jüdische Ärzte in Schöneberg / Topographie einer Vertreibung (Berlin 2012) 47-51

Jütte, Robert, «Die Vertreibung jüdischer und «staatsfeindlicher» Ärztinnen und Ärzte», in: ders. (Hrsg.), «Medizin und Nationalsozialismus. Bilanz und Perspektiven der Forschung» (Göttingen 2011) 83-93

Katz, Pearl, The Scalpel's Edge. The Culture of Surgeons (Boston, London, Toronto et al. 1999)

Keil, Dagmar, Die deutsche Hochschule für Leibesübungen in Berlin unter ihrem ersten Rektor August Bier (1920-1932), vorgestell anhand der Tätigkeitsberichte der Einrichtung (Med. Diss. Rostock 2006)

Kröner, Hans-Peter, «Die Emigration deutschsprachiger Mediziner im Nationalsozialismus», Berichte zur Wissenschaftsgeschichte 12 (1989) Sonderheft, *1_*44

Kuhn, Annette, Ich trage einen goldenen Stern. Ein Frauenleben in Deutschland (Berlin 2003)

Lammel, Hans-Uwe, «Tradition auf Abruf. Zur Personalpolitik an der Berliner Chirurgischen Universitätsklinik in der Ziegelstraße zwischen 1933 und 1945», in: Günter Grau/Peter Schneck (Hrsg.), Akademische Karrieren im «Dritten Reich». Beiträge zur Personal- und Berufungspolitik an Medizinischen Fakultäten (Berlin 1993) 63-75

Lammel, Hans-Uwe, «Chirurgie und Nationalsozialismus am Beispiel der Berliner Chirurgischen Universitätsklinik in der Ziegelstraße», in: Wolfram Fischer/Klaus Hierholzer/Michael Hubenstorf et al.: Exodus von Wissenschaften aus Berlin. Fragestellungen - Ergebnisse - Desiderate. Entwicklungen vor und nach 1933 (Berlin, New York 1994) 568-591

Lammel, Hans-Uwe, «August Bier und der Dritte Humanismus», in: K.-D. Fischer/ D. Nickel/P. Potter (Hrsg.), Text and Tradition. Studies in Ancient Medicine and its Transmission. Presented to Jutta Kollesch (Leiden, Boston, Köln 1998) 175-202 
Lorenz, Ina, «Die dunklen und schweren Jahre (1933-1934)», in: Israelitisches Krankenhaus Hamburg (Hrsg.), 150 Jahre Israelitisches Krankenhaus in Hamburg (Hamburg 1997) 65-85

Lorenz, Konrad, «Gottlob, unsere Nachkommen ... werden sich von den Tieren weiter entfernen als wir», Süddeutsche Zeitung 14./15./16.8.1964

Lorenz, Konrad, Das sogenannte Böse. Zur Naturgeschichte der Aggression (Wien 1963, München 1974)

Mannheim, Hans L., "80 years old", Bulletin of the Rudolf Virchow Medical Society in the City of New York (Anfang 1963; Abschrift in: Staatsarchiv Hamburg Bestand 611-11, Sig. 38 [2])

Marx, Gertie F., "The First Spinal Anesthesia. Who Deserves the Laurels?" Regional Anesthesia 19 (1994) 429-430

Moll, Friedrich H./Matthis Krischel/Peter Rathert et al., «Urologie und Nationalsozialismus. Paul Rosenstein 1875-1964 - zerrissene Biographie eines jüdischen Urologen», Urologe 50 (2011) 1143-1153

Pearle, Kathleen M., «Ärzteemigration nach 1933 in die USA: Der Fall New York» Medizinhistorisches Journal 19 (1984) 112-137

Pross, Christian, Wiedergutmachung. Der Kleinkrieg gegen die Opfer (Frankfurt am Main 1988; Berlin 22001)

van Rahden, Till, «Juden und die Ambivalenzen der bürgerlichen Gesellschaft in Deutschland von 1800 bis 1933», in: Ralph Jessen/Sven Reichardt/Ansgar Klein (Hrsg.),Zivilgesellschaft als Geschichte. Studien zum 19. und 20. Jahrhundert (Wiesbaden 2004) 345-369

Rosenstein, Paul, Narben bleiben zurück. Die Lebenserinnerungen des großen jüdischen Chirurgen (Bad Wörishofen 1954)

Rürup, Reinhard, «Jüdische Geschichte in Deutschland. Von der Emanzipation bis zur nationalsozialistischen Gewaltherrschaft», in: Dirk Balsius/Dan Diner (Hrsg.), Zerbrochene Geschichte. Leben und Selbstverständnis der Juden in Deutschland (Frankfurt am Main 1991) 79-102

Schagen, Udo, «Wer wurde vertrieben? Wie wenig wissen wir? Die Vertreibungen aus der Berliner Medizinischen Fakultät 1933. Ein Überblick», in: Sabine Scheiermacher/Udo Schagen, Die Charité im Dritten Reich. Zur Dienstbarkeit medizinischer Wissenschaft im Nationalsozialismus (Paderborn, München, Wien, Zürich 2008) 51-66

Schmiedebach, Heinz-Peter/Rebecca Schwoch, «Prof. Dr. med. Richard Hugo Georg Magnus (1883-1942)», in: Michael Sachs/Heinz-Peter Schmiedebach/Rebecca Schwoch, Deutsche Gesellschaft für Chirurgie 1933-1945. Die Präsidenten, hrsg. von Hans-Ulrich Steinau und Hartwig Bauer (Heidelberg 2011) 33-62

Schröder, Wilhelm Heinz, «Kollektive Biografien in der historischen Sozialforschung: Eine Einführung», in: Ders. (Hrsg.), Lebenslauf und Gesellschaft. Zum Einsatz von kollektiven Biografien in der historischen Sozialforschung (Stuttgart 1985) 7-17

Schwoch, Rebecca, Ärztliche Standespolitik im Nationalsozialismus. Julius Hadrich und Karl Haedenkamp als Beispiele (Husum 2001)

Schwoch, Rebecca (Hrsg.): Berliner jüdische Kassenärzte und ihr Schicksal im Nationalsozialismus. Ein Gedenkbuch (Berlin, Teetz 2009)

Schwoch, Rebecca, «Deutsche Gesellschaft für Chirurgie - Unsere verfolgten, vertriebenen und vermissten Mitglieder», in: Michael Sachs/Heinz-Peter Schmiede- 
bach/Rebecca Schwoch, Deutsche Gesellschaft für Chirurgie 1933-1945. Die Präsidenten, hrsg. von Hans-Ulrich Steinau und Hartwig Bauer (Heidelberg 2011) 219-225

Schwoch, Rebecca, «Medizinische Versorgung von Juden für Juden? Krankenbehandler> in Berlin 1938-1945», in: Caris-Petra Heidel (Hrsg.), Jüdische Medizin - Jüdisches in der Medizin - Medizin der Juden? (Frankfurt/Main 2011) 289-307

Taubert, Ernst, «100 Jahre Berliner Chirurgische Gesellschaft», in: Helmut Wolff (Hrsg.), Die Chirurgie vor 100 Jahren. Wissenschaftliches Symposium mit internationaler Beteiligung anlässlich des 100. Jahrestages der Gründung der Berliner Chirurgischen Gesellschaft und des 150. Geburtstages von Ernst v. Bergmann, Berlin 20. und 21. November 1986 (Berlin 1992) 13-40

Ullman, Walther Hans, «100 Jahre Rudolf Virchow Gesellschaft - in der Stadt New York», Medizinische Welt 1961, Nr. 1, 55-56

von Villiez, Anna, Mit aller Kraft verdrängt. Entrechtung und Verfolgung <nicht arischer> Ärzte in Hamburg 1933 bis 1945 (München, Hamburg 2009)

Vogeler, Karl, August Bier. Leben und Werk (München, Berlin 1941, ${ }^{2} 1942$ )

Vogeler, Karl, «Zum 100. Geburtstag von August Bier. 24. November 1961», Deutsche Medizinische Wochenschrift 86 (1961) 2554-2555

Vogeler, Karl, «August Biers Lebenskampf», Berliner Medizin 13 (1962) 325-329

Winau, Rolf, «James Israel», in: Wilhelm Treue/Rolf Winau (Hrsg.), Berlinische Lebensbilder II: Mediziner (Berlin 1987) 251-263

Winau, Rolf/Ekkehard Vaubel, Chirurgen in Berlin. 100 Porträts (Berlin, New York 1983)

Wollheim, Ernst, «Arthur Israel zum 80. Geburtstag», Münchener Medizinische Wochenschrift 13 (1963) 1818-1819 (= Wollheim 1963a)

Wollheim, Ernst, «Arthur Israel - achtzig Jahre», Aufbau 21.6.1963 (liegt vor in: Staatsarchiv Hamburg Bestand 611-11, Sig. 38 (2) (= Wollheim 1963b) 